BROWN-HET-934

DAMTP 94-5

March 1994

\title{
FORMATION OF TOPOLOGICAL DEFECTS IN A SECOND ORDER PHASE TRANSITION
}

\author{
Robert H. Brandenberger ${ }^{1)}$ and Anne-Christine Davis ${ }^{2)}$ \\ 1) Physics Department, Brown University, Providence, RI 02912, USA \\ 2) Department of Applied Mathematics and Theoretical Physics \\ and Kings College, University of Cambridge, Cambridge CB3 9EW, U.K.
}


The classical evolution equations of the Abelian Higgs model are studied at temperatures below the Ginsburg temperature of a phase transition which is assumed to be second order. It is shown that the initial thermal fluctuations provide a domain structure which is stable against late time fluctuations. This result lends support to the Kibble mechanism for the formation of topological defects. 


\section{Introduction}

Topological defects ${ }^{1}$ ) are playing an increasing role in various branches of physics. In particular, cosmic strings and global textures give rise to attractive scenarios for the origin of structure in the early Universe (for recent reviews see e.g., Refs. 2 and 3). It is therefore important to obtain a detailed understanding of the rate of formation of topological defects in phase transitions from a hot symmetric phase to a cold phase with broken symmetry.

The original mechanism of a defect formation is due to Kibble ${ }^{1)}$. He argued that at the phase transition, in any theory which admits topological defects, a network of such defects with correlation length (i.e., typical separation) $\xi$ will be frozen in at the Ginsburg temperature $T_{G}$. Here, $\xi$ is the correlation length at $t_{G}$, the cosmic time corresponding to temperature $T_{G}$.

Starting point of Kibble's argument was the assumption that on scales larger than $\xi$, the orientation of the order parameter in the vacuum manifold is random, that the order parameter smoothly interpolates between these random values, and that there thus is a finite probability to have nontrivial winding. This probability depends on the topology of the vacuum manifold and has been calculated in several interesting cases in Ref. 4.

The Kibble argument has been widely used in cosmology. For example, it has been used to generate the initial string configurations ${ }^{5)}$ for cosmic string evolution studies, to calculate the abundance of magnetic monopoles ${ }^{6)}$, and to justify the occurrence of textures ${ }^{7)}$ in models with nonvanishing $\pi_{3}(\mathcal{M}), \mathcal{M}$ being the vacuum manifold. Another application of the Kibble mechanism is the prediction of vortices in a pressure quench of superfluid helium ${ }^{8)}$.

The two main assumptions of the Kibble mechanism are that the order parameter takes on random values in $\mathcal{M}$ on scales larger than $\xi$, and that it smoothly interpolates between its values at different points in space (the "geodesic rule"). Recently ${ }^{9}$, the validity of the geodesic rule has been challenged, in particular 
for gauge theories. Since the energy density in gauge theories is proportional to $\left(D_{\mu} \phi\right)^{2}$ and not $\left(\partial_{\mu} \phi\right)^{2}$ (where $D_{\mu}$ is the covariant derivative operator), the order parameter $\phi$ need not interpolate smoothly in order to minimize the gradient energy. Hence, it has become important to investigate the validity of the Kibble mechanism more carefully.

For theories with a global symmetry, the geodesic rule is well justified. In this case, the Kibble mechanism has been tested both in numerical simulations ${ }^{10)}$ and in the laboratory ${ }^{11,12)}$.

For theories with a local symmetry the situation is less clear. In models with a first order phase transition, a recent analysis ${ }^{13)}$ of the dynamics of the classical fields has provided strong support for the Kibble mechanism. The main idea of the analysis, however, hinged on the phase transition proceeding via the nucleation and subsequent collision of bubbles of the broken symmetry phase. The methods are therefore not directly applicable to models with a second order phase transition.

In this letter we investigate the solution of the classical equations of motion for an Abelian Higgs model with a second order phase transition. We consider a field configuration set up by thermal fluctuations at the Ginsburg temperature and study its stability against thermal fluctuations present at later times. We conclude that the initial domain structure is preserved, although naturally the amplitude of the order parameter increases. Our results lend support to the hypothesis that the Kibble mechanism applies also to gauge theory defects produced in a second order transition. 


\section{System and Basic Equations}

As a toy model we consider the Abelian Higgs model with a complex scalar field $\phi$ and a $\mathrm{U}(1)$ gauge connection $A_{\mu}$. Its Lagrangean is

$$
\mathcal{L}=\left(D_{\mu} \phi\right)^{\dagger} D^{\mu} \phi-V(\phi)-\frac{1}{4} F_{\mu \nu} F^{\mu \nu}
$$

where

$$
D_{\mu}=\partial_{\mu}-i e A_{\mu}
$$

is the covariant derivative with gauge coupling constant $e, F_{\mu \nu}$ is the field strength tensor, and $V(\phi)$ is the potential for $\phi$. The potential is chosen such that phase transition is second order.

In Lorentz gauge

$$
\partial_{\mu} A^{\mu}=0
$$

the equations of motion for $\phi$ and $A_{\mu}$ become

$$
\left(\partial_{\mu} \partial^{\mu}-2 i e A_{\mu} \partial^{\mu}-e^{2} A_{\mu} A^{\mu}\right) \phi+2 \frac{\partial V}{\partial|\phi|^{2}} \phi=0
$$

and

$$
\partial_{\mu} \partial^{\mu} A_{\nu}-2 e^{2} A_{\nu}|\phi|^{2}=-i e \phi^{*} \vec{\partial}_{\nu} \phi
$$

It is convenient to separate Eq. (2.4) into equations for the amplitude $\rho$ and phase $\alpha$ of $\phi$. Inserting

$$
\phi=\rho e^{i \alpha}
$$

into (2.4) we obtain

$$
\partial^{2} \rho-(\partial \alpha-e A)^{2} \rho-e^{2} A^{2} \rho+2 \frac{\partial V}{\partial \rho^{2}} \rho=0
$$


and

$$
\partial^{2} \alpha+2\left(\partial^{\mu} \alpha-e A^{\mu}\right) \partial_{\mu} \rho \frac{1}{\rho}=0 .
$$

We wish to study the evolution of the solutions of the coupled system (2.5), (2.7) and (2.8) of differential equations during a second order phase transition. We assume the following form for the finite temperature effective potential

$$
V_{T}(\phi)=\frac{1}{4} \lambda|\phi|^{4}-\frac{1}{2}\left(\lambda \eta^{2}-\tilde{\lambda} T^{2}\right)|\phi|^{2}+\frac{1}{4} \lambda \eta^{4} .
$$

Here, $\lambda$ is the self-coupling constant of $\phi, \tilde{\lambda}$ is a coupling constant determined by the graphs which dominate the one loop effective potential, and $\eta$ is the value of $|\phi|$ in the vacuum manifold (the scale of symmetry breaking). In the following we take $\tilde{\lambda} \simeq \lambda . T$ is the temperature.

The phase transition proceeds by spinodal decomposition ${ }^{14)}$. Due to thermal fluctuations in the initial state, the phase $\alpha$ will take on random values on scales larger than the correlation length $\xi$. The temperature dependence of $\xi$ can be obtained by equating spatial gradient energy required to set up the domains and potential energy gain by having $\phi$ deviate from 0 . For temperatures below the critical temperature

$$
T_{c}=\lambda^{1 / 2} \tilde{\lambda}^{-1 / 2} \eta \simeq \eta,
$$

the result for $\xi(T)$ is ${ }^{1)}$

$$
\xi(T) \simeq \lambda^{-1 / 2} \eta^{-1}\left(1-\left(\frac{T}{T_{c}}\right)^{2}\right)^{-1 / 2}
$$

As long as the temperature is higher than the Ginsburg temperature $T_{G}$, these domains are unstable to thermal fluctuations. The value of $T_{G}<T_{c}$ is determined by equating thermal energy with the energy of the domains determined above. The 
result is

$$
\xi\left(T_{G}\right) \simeq \lambda^{-1} T_{G}^{-1} \simeq \lambda^{-1} \eta^{-1}
$$

These qualitative arguments suggest that below $T_{G}$, the domain structure is frozen in. Since the winding number $n_{\gamma}$ around a closed curve $\gamma$ is

$$
n_{\gamma}=-\frac{i}{4 \pi} \oint \frac{\phi^{* \leftrightarrow} \partial_{\mu} \phi}{|\phi|^{2}} d s^{\mu}
$$

the random distribution of phases will induce a finite probability to have a topological defect (in our case a cosmic string) in any correlation volume $\xi\left(T_{G}\right)^{3}$.

To reach the final conclusion, we implicitly made use of the "geodesic rule" which says that along a curve $\gamma$ connecting two domains, the phase $\alpha$ will smoothly interpolate between the values of $\alpha$ in the two pluses. This rule can be justified in a theory with global symmetry (since it comes from minimizing the spatial gradient energy), but not in a gauge theory (since the gauge fields can compensate large scalar field gradients).

In previous work ${ }^{13)}$, we studied defect formation in a gauge theory with a first order phase transition. In this case, the phase transition proceeds by nucleation of true vacuum bubbles $(|\phi| \sim \eta, \alpha$ constant) in a surrounding sea of false vacuum $(\phi=0)$. Using the equations of motion to study the evolution of $\phi$ when bubbles collide, we were able to show that the geodesic rule is dynamically realized.

In the case of a second order transition we must use rather different methods. We will again argue that the geodesic rule is dynamically realized. The basic logic is as follows: For $T>T_{G}$, thermal fluctuations will dominate the field configurations for $\phi$ and $A_{\mu}$. We can picture all fields as superpositions of plane waves with uncorrelated phases. Fluctuations with wavelength $\xi(T)$ will dominate. Those with $\xi<\xi(T)$ are energetically suppressed while those with $\xi>\xi(T)$ are phase space suppressed.

We will therefore study an initial field configuration with typical wavelength $\xi(T)$ for which the geodesic rule is obviously satisfied. We shall argue - using the 
dynamical equations (2.5), (2.7) and (2.8) - that the induced domain structure is stable for $T \leq T_{G}$ : the change in the phase induced by the dynamics for $T<$ $T_{G}$ is subdominant, and thermal fluctuations at $T=T_{G}$ do not lead to a large perturbation from the evolution of the amplitude $\rho$ in the absence of fluctuations.

Note that our analysis is done in a particular gauge. However, the final conclusion concerning the nonvanishing probability for nonzero winding number is gauge independent.

\section{Perturbation Analysis}

In the following we study the initial stages of spinodal decomposition when $\rho \ll \eta$. We consider as initial field configuration at $T=T_{G}$ a plane wave $\rho_{0}(\underline{x}), \alpha_{0}(\underline{x}), A_{0}(\underline{x})$ with wave number $k$ (the phases of the three fields are arbitrary). In the absence of fluctuations this background configuration will evolve smoothly in time preserving the initial domain structure and winding number.

Our main goal is to study the stability of this configuration (and its winding number) against thermal fluctuations. The coupling to thermal fluctuations can be modelled ${ }^{15)}$ by introducing a second scalar field $\psi$ which is assumed to remain in thermal equilibrium and which is coupled to $\phi$ via the interaction Lagrangean

$$
\mathcal{L}_{I}=\frac{1}{2} g \psi^{2}|\phi|^{2}
$$

leading to a source term $-g \psi^{2} \rho$ on the right hand side of (2.7).

The effects of thermal fluctuations are analyzed to first order in a perturbative expansion about $\left(\rho_{0}, \alpha_{0}, A_{0}\right)$. The total field configurations are

$$
\begin{aligned}
\rho(\underline{x}, t) & =\rho_{0}(\underline{x}, t)+\rho_{I}(\underline{x}, t) \\
\alpha(\underline{x}, t) & =\alpha_{0}(\underline{x}, t)+\alpha_{I}(\underline{x}, t) \\
A(\underline{x}, t) & =A_{0}(\underline{x}, t)+A_{I}(\underline{x}, t),
\end{aligned}
$$

and the equations of motion are expanded to first order in $\rho_{I}, \alpha_{I}, A_{I}$ and $g$. All perturbations are induced by the coupling to the thermal bath and hence will be 
of order $g$. Our goal is to show that the perturbations remain smaller than the background solution provided that $k^{-1}$ corresponds to the Ginsburg length $\xi\left(T_{G}\right)$.

The perturbation equations are

$$
\begin{aligned}
\left(\partial_{t}^{2}-\nabla^{2}\right) \rho_{I} & -\left[\dot{\alpha}_{0}^{2}-\left(\nabla \alpha_{0}\right)^{2}-2 \frac{\partial V}{\partial \rho^{2}}+2 \frac{\partial \partial V}{\partial \rho \partial \rho^{2}} \rho_{0}\right] \rho_{I} \\
& =\left[-g \psi^{2}+\dot{\alpha}_{0} \dot{\alpha}_{I}-\nabla \alpha_{0} \nabla \alpha_{I}\right] \rho_{0}
\end{aligned}
$$

and

$$
\left(\partial_{t}^{2}-\nabla^{2}\right) \alpha_{I}+2 \partial^{\mu} \alpha_{I} \partial_{\mu} \rho_{0} \frac{1}{\rho_{0}}+2 \partial^{\mu} \alpha_{0} \partial_{\mu} \rho_{I} \frac{1}{\rho_{0}}-2 \partial^{\mu} \alpha_{0} \partial_{\mu} \rho_{0} \frac{\rho_{I}}{\rho_{0}^{2}}=0
$$

where the gauge fields have been set to zero to simplify the equations (they will be included later). The perturbations vanish at the initial time $t=t_{G}$.

For $k<\lambda^{1 / 2} \eta=k_{\text {crit }}$ the background equation for $\rho_{0}$ has an exponential instability

$$
\partial_{t}^{2} \rho_{0} \simeq m_{I}^{2} \rho_{0} \simeq\left(\lambda \eta^{2}-k^{2} \alpha_{0}\right) \rho_{0} \simeq \lambda \eta^{2} \rho_{0}
$$

with

$$
\rho_{0}(\underline{x}, t) \sim e^{m_{I}\left(t-t_{G}\right)}, m_{I} \simeq \lambda^{1 / 2} \eta
$$

The solution for the phase $\alpha_{0}$ can be taken to be time independent with spatial gradient proportional to $k$.

The basic logic of our analysis is as follows: we first analyze the equation of motion (3.3) for $\rho_{I}$ under the assumption that the length scale of fluctuations in $\alpha_{I}$ is the same as for those in $\rho_{I}$. Using the Green's function method it can be shown that

$$
\frac{\rho_{I}}{\rho_{0}}<1 \text { for } k_{p}<k_{\text {crit }}
$$

where $k_{p}$ is the wave number for the perturbation. Next, we consider the equation of motion (3.4) for $\alpha_{I}$ and show that modes of $\alpha_{I}$ do not grow in time for 
wavenumbers larger than $k$. This shows that the assumption about the length scale of fluctuations in $\alpha_{I}$ in the first step of the analysis was self-consistent. In a final step we demonstrate that the prescence of gauge fields does not invalidate the previous considerations. Now the details:

Picking out the dominant terms based on the above considerations, Eq. (3.3) becomes

$$
\left(\partial_{t}^{2}-\nabla^{2}\right) \rho_{I}-m_{I}^{2} \rho_{I}=\left[-g \psi^{2}-\nabla \alpha_{0} \nabla \alpha_{I}\right] \rho_{0} \simeq-g \psi^{2} \rho_{0}
$$

with the same $m_{I}$ as in the background equation (3.5). This equation can be solved using the Green function method, i.e.,

$$
\rho_{I}(\underline{x}, t)=-g \int_{0}^{t} d \tau d^{3} y G_{r e t}(t-\tau, \underline{x}-\underline{y}) \psi^{2}(\tau, \underline{y}) \rho_{0}(\tau, \underline{y}),
$$

where $G_{r e t}$ is the retarded Green function. A new time variable has been chosen such that $t=0$ is the onset of spinodal decomposition when $\rho_{I}=0$.

In order to estimate the right hand side of (3.9) we can replace $\rho_{0}(\tau, \underline{y})$ by its upper bound

$$
\rho_{0}(\tau, \underline{y})<\mathcal{A}_{0} e^{m_{I} \tau},
$$

where $\mathcal{A}_{0}$ is the initial amplitude. If the phase transition is rapid, we can drop the $\tau$ dependence of the thermal source $\psi^{2}(\tau, \underline{y})$. Inserting the form of the retarded Green function

$$
G_{r e t}(x)=-\frac{1}{(2 \pi)^{4}} \int d^{4} p \frac{e^{-i p x}}{\left(p_{0}+i \varepsilon\right)^{2}-\underline{p}^{2}+m_{I}^{2}}
$$

we obtain

$$
\rho_{I}(t, \underline{x}) \sim \frac{g \mathcal{A}_{0}}{(2 \pi)^{4}} \int d^{3} p e^{i p \cdot x} \tilde{\psi}^{2}(\underline{p}) \int_{-\infty}^{\infty} d p_{0} e^{-i p_{0} t} \int_{0}^{t} d \tau \frac{e^{\left(m_{I}+i p_{0}\right) \tau}}{\left(p_{0}+i \varepsilon\right)^{2}-\underline{p}^{2}+m_{I}^{2}} .
$$

The $\tau$ integral can be done explicitly. At large $p_{0}$, the resulting integral scales as $p_{0}^{-3}$. Hence, the integral over $p_{0}$ can be written as an integral over a closed contour 
and solved using the method of residues. It is easy to consider the cases $|p|<m_{I}$ and $|p|>m_{I}$ separately. In both cases, the integral is bounded by $\pi / m_{I}^{2}$. Hence, our estimate for (3.12) is

$$
\rho_{I}(t, \underline{x}) \sim \frac{g}{2 \pi} \mathcal{A}_{0} e^{m_{I} t} \frac{\pi}{m_{I}^{2}} \psi^{2}(\underline{x})
$$

The amplitude of $\psi^{2}(\underline{x})$ is determined by thermal equilibrium. If $\psi$ is a self interacting scalar field with self coupling constant of the order 1 , then

$$
\psi^{2}(\underline{x}) \sim T^{2}
$$

Hence, the ratio of $\rho_{I}$ to $\rho_{0}$ is

$$
\frac{\rho_{I}}{\rho_{0}} \sim g \frac{T^{2}}{m_{I}^{2}}
$$

At the onset of spinodal decomposition

$$
T=T_{G}<T_{c}=\lambda^{1 / 2} \tilde{\lambda}^{-1 / 2} \eta=\lambda^{1 / 2} g^{-1 / 2} \eta
$$

(see (2.10)), and hence

$$
\frac{\rho_{I}}{\rho_{0}}<1
$$

We conclude that thermal fluctuations do not dominate the evolution of $\rho$ for $T<T_{c}$ (they do dominate at higher temperatures!) However, the derivation makes use of two key assumptions which must be shown to be at least self consistent with the above analysis. Firstly, it is assumed that the wave number of fluctuations in $\alpha_{I}$ are smaller than $k$. Otherwise, the second source term in (3.8) (proportional to $\left.\nabla \alpha_{0} \nabla \alpha_{I}\right)$ might dominate. Secondly, it must be shown that the presence of gauge fields does not disrupt the smooth evolution of $\rho$ and $\alpha$. 
First, we analyze the evolution of $\alpha(t, \underline{x})$. The initial field configuration $\rho_{0}(0, \underline{x}), \alpha_{0}(0, \underline{x})$ is assumed to have nonvanishing winding number along a given circle $C$ in space. For winding number 1 , the phase $\alpha_{0}(0, \underline{x})$ increases smoothly from 0 to $2 \pi$ along $C$. The only way the winding can disappear is for a discontinuity in $\alpha(\underline{x})$ to develop. This is only possible if at some point along $C$ the modulus $\rho$ vanishes. As shown above this is not possible as long as $\alpha_{I}$ does not develop small wavelength excitations.

We thus analyze the equation (3.4) for $\alpha_{I}$ under the assumption that $\rho_{I} / \rho_{0}<1$ in which case the last term on the left hand side of the equation is negligible. We approximate the second term as $2 i k_{\mu} \partial^{\mu} \alpha_{I}$. In Fourier space, the resulting equation is

$$
\left(\partial_{t}^{2}+k_{I}^{2}-2 k \cdot k_{I}\right) \tilde{\alpha}_{I}=2 k \cdot k_{I} \frac{\tilde{\rho}_{I}}{\rho_{I}}
$$

where tilde signs denote the Fourier modes labelled by wave vector $\underline{k}_{I}$. This inhomogeneous equation can once again be solved by the Green function method. For $k_{I} \gg k$ the solution is

$$
\tilde{\alpha}_{I}\left(k_{I}, \tau\right) \sim \frac{k \cdot k_{I}}{k_{I}^{2}} \frac{\tilde{\rho}_{I}\left(k_{I}\right)}{\rho},
$$

which shown that short wavelength fluctuations are suppressed compared to long wavelength inhomogeneities.

The arguments which lead to (3.17) and (3.19) are self consistent. We could also reverse the logic and first study the $\alpha$ equation under the assumption $\rho_{I} / \rho_{0}<$ 1 , with the result that fluctuations of $\alpha$ on wavelengths smaller than $k^{-1}$ are suppressed. This result could then be used in the $\rho$ equation to verify that indeed $\rho_{I} / \rho_{0}<1$.

All the previous arguments neglect the presence of gauge fields and are thus only applicable to global symmetries and global defect formation. We now demonstrate that the inclusion of gauge fields (working in Coulomb gauge) does not invalidate the main conclusions. 
In the presence of gauge fields, the perturbation equation (3.3) reads

$$
\begin{aligned}
\partial^{2} \rho_{I} & -\left[\left(\partial \alpha_{0}-e A_{0}\right)^{2}+e^{2} A_{0}^{2}-2 \frac{\partial V}{\partial \rho^{2}}+2 \frac{\partial}{\partial \rho} \frac{\partial V}{\partial \rho^{2}} \rho_{0}\right] \rho_{I} \\
& =\left[-g \psi^{2}+e^{2} A_{0} A_{I}+\left(\partial \alpha_{0}-e A_{0}\right)\left(\partial \alpha_{I}-\partial A_{I}\right)\right] \rho_{0} .
\end{aligned}
$$

At the beginning of spinodal decomposition $\left(T=T_{G}\right)$, the amplitude of $A_{0}=0$ is constrained by thermal equilibrium considerations. Note that the initial configuration at $T_{G}$ will typically contain magnetic fields with coherence length $\xi\left(T_{G}\right)^{16)}$. Hence, it is invalid to set $A_{0}=0$ as initial configuration. However, since

$$
\left|D_{\mu} \psi D^{\mu} \psi\right|<T^{4}
$$

and $\psi \sim T$, it follows that $A$ is bounded by

$$
A<e^{-1} T
$$

Inserting this bound in (3.20) we conclude that the terms involving gauge fields are negligible provided

$$
T<\lambda^{1 / 2} \eta \equiv T_{A}
$$

We suspect that improved estimates would show that this "critical" temperature is in fact $T_{G}$.

In the presence of nonvanishing gauge fields, Eq. (3.4) is modified by

$$
\partial^{\mu} \alpha_{0} \rightarrow \partial^{\mu} \alpha_{0}-e A_{0}^{\mu}
$$

and by the presence of an extra source term $e A_{I}^{\mu} \frac{\partial_{\mu} \rho_{0}}{\rho_{0}}$. However, even at $T=T_{G}$ the new terms are of the same order of magnitude or smaller than the ones present in (3.4). Hence, the previous conclusions concerning the absence of short wavelength inhomogeneities in $\alpha_{I}$ remain true.

In summary, we have shown that even in the presence of gauge fields, the initial domain structure and winding numbers present at $T=T_{A}$ are frozen in. 


\section{Discussion}

In this paper we have studied the classical dynamics of spinodal decomposition in the Abelian Higgs model coupled to a thermal bath. We considered the classical evolution of an initial field configuration at the Ginsburg temperature which was assumed to have nontrivial domain structure on a length scale $\xi\left(T_{G}\right)$ (and hence a finite probability to have nonvanishing winding number). We argued that the initial domain structure is stable against thermal fluctuations. Hence, the winding number is preserved. Our results lend support to the Kibble mechanism for defect formation - for both global and local defects.

Above the Ginsburg temperature, thermal fluctuations dominate. Configurations with nonvanishing winding number are created and destroyed with a frequency determined by thermal equilibrium. Our results indicate that this domain structure created by thermal fluctuations freezes out at $T_{G}$, leaving behind a field configuration with finite probability for nonvanishing winding number on the scale $\xi\left(T_{G}\right)$.

Our analysis is unfortunately rather qualitative. It is based on approximate equations. We introduced thermal fluctuations by a coupling to an additional scalar field, but we did not introduce the dissipation term which must be present in order to be in concordance with the fluctuation-dissipation theorem. However, such dissipation terms would only slow the growth of fluctuations and would hence only strengthen our conclusions.

The analysis of the equations presented in this paper is only approximate. Only the initial stages of spinodal decomposition in which most nonlinearities are negligible were analyzed. Even this analysis was done in an approximate way.

Our classical analysis can be improved by evolving the solutions of the equations of motion numerically. A second improvement would be to study the Liouville equation associated with our set of classical equations and to derive a result for

$P\left(\varphi_{i}, \dot{\varphi}_{i}, t\right)$, the probability distribution of the fields ${ }^{15)}$. Here, $\varphi_{i}$ stands for the 
collection of all fields in the problem. Finally, the analysis should be extended to a semiclassical one to control the effects of quantum fluctuations near the onset of spinodal decomposition. Work on these projects is in progress.

$\underline{\text { Acknowledgements: }}$

We are grateful to Mark Hindmarsh for many discussions about the issues discussed here. We also thank Ed Copeland, Tom Kibble and Ajit Srivastava for interesting conversations. This work is supported in part by the US Department of Energy under contract DE-FG02-91ER40688, Task A, and by an NSF-SERC collaborative research award NSF-INT-9022895 and SERC GR/G37149.

\section{REFERENCES}

1. T.W.B. Kibble, J. Phys. A9, 1387 (1976);

T.W.B. Kibble, Phys. Rep. 67, 183 (1980).

2. R. Brandenberger, Phy. Scripta T36, 114 (1991);

N. Turok, Phys. Scripta T36, 135 (1991).

3. A. Vilenkin and E.P.S. Shellard, 'Cosmic Strings and Other Topological Defects' (Cambridge Univ. Press, Cambridge, 1994).

4. T. Prokopec, Phys. Lett. B262, 215 (1991);

R. Leese and T. Prokopec, Phys. Rev. D44, 3749 (1991).

5. T. Vachaspati and A. Vilenkin, Phys. Rev. D30, 2036 (1984);

A. Albrecht and N. Turok, Phys. Rev. Lett. 54, 1868 (1985).

6. J. Preskill, Phys. Rev. Lett. 43, 1365 (1979);

Ya. B. Zel'dovich and M. Khlopov, Phys. Lett. B79, 239 (1978).

7. N. Turok, Phys. Rev. Lett. 63, 2625 (1989).

8. W. Zurek, Nature 317, 505 (1985).

9. S. Rudaz and A. Srivastava, Mod. Phys. Lett. A8, 1443 (1993). 
10. J. Ye and R. Brandenberger, Mod. Phys. Lett. A5, 157 (1990).

11. I. Chuang, R. Durrer, N. Turok and B. Yurke, Science, 251, 157 (1991);

B. Yurke, A. Pargellis, I. Chuang and N. Turok, Physica B178, 56 (1992);

M. Bowick, L. Chandar, E. Schiff and A. Srivastava, Science 16, 943 (1994).

12. P. Hendry, et al., J. Low Temp. Phys. 93, 1059 (1993).

13. M. Hindmarsh, A.-C. Davis and R. Brandenberger, Phys. Rev. D, in press (1994).

14. J. Langer, Physica 73, 61 (1974).

15. R. Brandenberger, H. Feldman and J. MacGibbon, Phys. Rev. D37, 2071 (1987).

16. T. Vachaspati, Phys. Lett. B265, 258 (1991). 